\title{
CARDIOVASCULAR ABNORMALITIES IN PATIENTS WITH HIV INFECTION: A BOLT IN BLUE
}

\author{
Anita Arya ${ }^{1}$, Ashish Deshmukh², Kaustubh Dilip Mundada ${ }^{3}$ \\ ${ }^{1}$ Associate Professor, Department of Medicine, Gandhi Medical College, Bhopal. \\ ${ }^{2}$ Senior Resident, Department of Medicine, Gandhi Medical College, Bhopal. \\ 33rd Year Resident, Department of Medicine, Gandhi Medical College, Bhopal.
}

\section{ABSTRACT}

One of the consistent findings among various studies on HIV has been simultaneous multiorgan dysfunction. Cardiovascular disorders are now most common cause of mortality worldwide. With more effective and widespread treatment of HIV in resourcerich settings, morbidity and mortality from non-AIDS-related events have surpassed those from AIDS-related events with cardiovascular diseases emerging as an important cause of death in HIV-infected patients relative to the decreasing incidence of opportunistic disease. Various studies have reported a 1.5-fold increase in the rate of cardiovascular events in HIV-infected individuals compared to control populations.

\section{MATERIAL AND METHODS}

The aim of the study was to the find the prevalence and types of different cardiovascular abnormalities in HIV positive patients and assess their association with CD4 counts. Consecutive 82 patients, HIV positive patients fulfilling the inclusion criteria and giving informed consent were included in the study. All patients were subjected to history taking and a detailed physical examination. Blood counts, renal function tests, lipid profile and CD4 counts were estimated and patients were subjected to 12-lead ECG, chest X-ray and 2D/Colour Doppler Echocardiogram.

\section{RESULTS}

Of the 82 patients studied $47.46 \%$ had evidence of cardiovascular involvement, out of which $12 \%$ had clinical features of heart failure while electrocardiographic changes were seen in 35\% of patients in the form of sinus tachycardia (27\%), QTc prolongation $(10 \%)$ and left sided chamber enlargements (6\%). Echocardiographic abnormalities were noted in 39 patients (47.56\%) including fractional shortening associated with systolic dysfunction $(26.8 \%)$. The mean CD4 count in patients with echocardiographic abnormalities was found to be $58.87 \pm 29.80$, whereas in patients without echocardiographic abnormalities it was $136.53 \pm 38.80$ $(\mathrm{p}<0.0001)$.

\section{CONCLUSION}

High frequencies of cardiac abnormalities, both symptomatic and asymptomatic were detected in the HIV/AIDS patients in our study. Furthermore, the study concluded that a careful initial and periodic cardiac evaluation to detect early involvement of the cardiovascular system in the HIV disease is recommended. Since the HIV-infected population is relatively young and actual cardiovascular events are infrequent, more long terms studies are needed.

\section{KEYWORDS}

HIV, CD4 Counts, Cardiovascular Abnormalities.

HOW TO CITE THIS ARTICLE: Anita Arya, Ashish Deshmukh, Kaustubh Dilip Mundada. "Cardiovascular Abnormalities in Patients with HIV Infection: A Bolt in Blue." Journal of Evolution of Medical and Dental Sciences 2015; Vol. 4, Issue 99, December 10; Page: 16395-16399, DOI: $10.14260 /$ jemds/2015/2427

\section{INTRODUCTION}

HIV/AIDS is a multisystemic disease, affecting virtually every organ and system of the body and causing progressive dysfunction. Cardiac involvement impacts on the natural history and prognosis of the HIV disease. Of interest is the observation that the incidence of AIDS-related heart disease found in post-mortem studies is significantly higher than the incidence of abnormalities diagnosed clinically ante-mortem. Therefore, it is possible that many AIDS patients have cardiac abnormalities that are not recognized during the course of their illness.

Financial or Other, Competing Interest: None.

Submission 16-11-2015, Peer Review 17-11-2015,

Acceptance 02-12-2015, Published 08-12-2015.

Corresponding Author:

Dr. Kaustubh Dilip Mundada,

Room No. 72, F-Block,

Gandhi Medical College Hostel,

Bhopal-462001.

E-mail: kaustubh2013@gmail.com

DOI: $10.14260 /$ jemds $/ 2015 / 2427$
In an autopsy study carried out in In an autopsy study carried out in 1998, cardiac abnormalities were noted in twothirds of the patients with AIDS. These abnormalities, which were attributed directly or indirectly to the HIV virus and/or treatment side effects, could largely have been detected early ante-mortem using echocardiography, a non-invasive radiation-free investigation.

Despite the availability of many single centre and multicentre reports a clear picture of how cardiovascular disease manifests in patients with HIV/AIDS is confounded in most available studies coming from developed countries by the presence of several comorbidities and prolonged history of drug intake. Considering the ever increasing population of HIV positives in our country and the significant causal relationship with cardiovascular abnormalities established by current literature, this study was undertaken to identify cardiovascular abnormalities in treatment-naïve patients in order to assess the cardiac effects of HIV infection while excluding drug effect. 


\section{MATERIAL AND METHODS}

This prospective study was conducted among HIV patients reporting to Anti Retroviral Therapy Center of Gandhi Medical College and associated Hamidia Hospital, Bhopal. The aim of the study was to find the incidence and types of cardiovascular abnormalities in HIV infection, to study the association between cardiovascular abnormalities and CD4 count. Inclusion criteria for the study were HIV-infected patients whose age more than 15 years, who are antiretroviral therapy (ART)-naïve and without any history or examination suggestive of ischemic, rheumatic, congenital, diabetic or hypertensive heart disease.

Eighty two HIV positive patients presenting to ART centre and fulfilling the above criteria were included in this study. All patients were subjected to a detailed history taking and a thorough physical examination. The CD4 counts were estimated along with other routine laboratory investigations like complete blood counts, renal function tests, lipid profile and fasting blood sugars. Patients then underwent screening with chest x-ray, 12-lead ECG and 2D and Color Doppler Echocardiogram.

\section{RESULTS}

A total of 82 HIV positive patients were studied, of which 63 were males and 19 females. The age ranged from 22 to 56 yrs. and the mean age was 34 yrs. Out of these 82 patients, 39 i.e. $47.56 \%$ had evidence of cardiovascular abnormalities. Most common cardiovascular abnormality found was systolic dysfunction 22(26.8\%), followed by pericardial effusion $17(21 \%)$, diastolic dysfunction $8(10 \%)$ and dilated cardiomyopathy with global hypokinesia 5(6\%), infective endocarditis $1(1.2 \%)$. The predominant mode of presentation was dyspnea; $43 \%$ of patients had evidence of cardiovascular involvement in the form of ECG changes as depicted in Fig 1.

Echocardiographic abnormalities were noted in 39 patients (47.56\%). As shown in Fig 2, most common echocardiographic abnormality found was reduction in fractional shortening associated with systolic dysfunction $(n=22,26.8 \%)$ and dilated cardiomyopathy $(n=5,6 \%)$ followed by left ventricular diastolic dysfunction (8 patients, $10 \%$ ), and pericardial effusion (17 patients, $21 \%$ ). While the pericardial effusions noted were small and asymptomatic, all patients with left ventricular diastolic dysfunction $8(10 \%)$ had Grade 1 diastolic dysfunction. Many of the patients presented with multiple abnormalities, which were shown in Fig 2.

Increased left atrial dimension $(>40 \mathrm{~mm})$ is seen in 5 (6\%) of patients. The mean left atrial dimension for patients having LV dysfunction and those without LV dysfunction is $33.63 \mathrm{~mm}$ and $29.8 \mathrm{~mm}$ respectively. Also increased RVD was seen in $6(7 \%)$ patients.

Increased left ventricular end diastolic dimension $(>56 \mathrm{~mm})$ is seen in $5(6 \%)$ patients. The mean left ventricular end diastolic dimension for patients having $\mathrm{LV}$ dysfunction and those without LV dysfunction is $50.51 \mathrm{~mm}$ and $42.55 \mathrm{~mm}$ respectively.

Increased left ventricular end systolic dimension ( $>39 \mathrm{~mm}$ ) is seen in $12(14 \%)$ patients with LV dysfunction. The mean left ventricular end systolic dimension for patients having LV dysfunction and those without LV dysfunction is $37.52 \mathrm{~mm}$ and $27.97 \mathrm{~mm}$ respectively. Diastolic dysfunction was present in $8(10 \%)$ patients, which was grade one in all these patients. These patients had E/A ratio less than 1.
In the present study, left ventricular ejection fraction was less than $55 \%$ in $22(26.8 \%)$ of patients. The mean EF for patients having $\mathrm{LV}$ dysfunction and those without LV dysfunction was $43.68 \pm 4.10 \%$ and $64.38 \pm 3.48 \%$ respectively. Fractional shortening less than $28 \%$ was seen in $22(26.8 \%)$ patients. The mean fractional shortening for patients having $\mathrm{LV}$ dysfunction and those without LV dysfunction are $22.29 \%$ and $33.86 \%$ respectively. The range was $30.76 \pm 0.77 \%$.

CD4 count was less than 100 per cumm in 26(31.7\%) patients having echocardiographic abnormalities; $13(12 \%)$ patients having echocardiographic abnormalities had CD4 count more than 100 per cumm; 31(37\%) patients without echocardiographic abnormalities had CD 4 count less than 100 per cumm while $12(14.6 \%)$ patients had CD4 count more than 100 per cumm. Mean CD4 count in patients with echocardiographic abnormalities was found to be $58.87 \pm 29.80$, whereas in patients without echocardiographic abnormalities it was $136.53 \pm 38.80(\mathrm{P}<.001)$ (Table 1$)$.

\section{DISCUSSION}

The degree to which HIV infection itself, traditional cardiovascular risk factors and ART each contribute to the elevated risk of cardiovascular disease in the HIV-infected population are unknown. In our study, we included only those patients who were not on antiretroviral therapy and found that the prevalence of cardiac abnormalities to be $47.56 \%$, which is far higher than previous studies evaluating this by P Aggrawal et al. but quite close to $50 \%$ prevalence seen in study by Hakim et al. In our study, we also found that patients with CD4 count $<100 / \mathrm{mm} 3$ had a high prevalence of cardiovascular abnormalities than those with CD 4 counts $>100 / \mathrm{mm} 3$, which was similar to study done by Klein D et al. ${ }^{1}$

With more effective and widespread treatment of HIV in resource-rich settings, morbidity and mortality from nonAIDS-related events have surpassed those from AIDS-related events. ${ }^{2-4}$ In particular, cardiovascular disease has emerged as an important cause of death in HIV-infected patients relative to the decreasing incidence of opportunistic disease.

Various studies have consistently reported a 1.5 -fold increase in the rate of cardiovascular events in HIV-infected individuals compared to control populations, although some of these studies are limited by low number of events, short follow-up and incomplete assessments of other cardiac risk factors. ${ }^{5-9}$ Overall, the classic cardiovascular risk factors of dyslipidemia, hypertension, diabetes and smoking are common among HIV-infected populations, although the frequency of these comorbidities is not sufficient to explain the overall increased incidence of cardiovascular disease observed in the setting of HIV infection.

This higher risk of CVD in HIV-infected patients than the general population originates from the development of atherosclerosis, which seems to be multifactorial in origin, related to the higher rates of smoking, dyslipidemia and other traditional risk factors in the HIV-infected population and the virus itself causing a chronic inflammatory state, which leads to endothelial dysfunction and atherosclerosis and also seems to be related to cART and the biological changes it cause. Compared with uninfected controls, HIV-infected individuals more frequently have low high-density lipoprotein cholesterol (HDL-c) and elevated triglycerides. Despite possible contributions of some ART drugs (Most studies implicate old protease inhibitors and this does not seem like a class effect). 
On cardiovascular risk, several studies suggest that higher CD4 cell counts and lower HIV RNA levels are associated with decreased MI risk.10,11,12 Discontinuation of ART is associated with an even greater risk of cardiovascular events suggesting a protective effect of suppression of HIV replication. ${ }^{13}$

Premature coronary artery pathology has been reported among HIV-positive individuals. Autopsy studies first suggested an association between vascular endothelial pathology and HIV. ${ }^{14}$ Reporting of clinically evident Coronary Artery Disease (CAD) has increased since the introduction of PIs in 1996. HIV infection was associated with a greater risk of acute MI overall, even after adjusting for Framingham risk factors and other comorbidities (Adjusted HR 1.48, 95\% CI 1.27-1.72). ${ }^{15}$

HIV infected patients have higher rates of MI than nonHIV infected patients, 11.13 versus 6.98 per 1000 personyears in a recent US study. ${ }^{16} \mathrm{~A}$ recent Danish study found that HIV infected patients receiving Highly Active Antiretroviral Therapy (HAART) were more likely to be hospitalized with ischemic heart disease than HIV-uninfected controls. ${ }^{17}$ Similarly, a Kaiser Permanente study found a higher rate of cardiovascular events in HIV infected patients who were not receiving ARV therapy compared to HIV uninfected controls. ${ }^{18}$

Left ventricular dysfunction, dilated cardiomyopathy, and myocarditis all occur with increased frequency in AIDS patients. In a pre-HAART study, global LV hypokinesis was found in $14.5 \%$ of patients and was associated with lower CD4 counts and congestive heart failure was diagnosed in about $2 \%$ of patients. ${ }^{19}$ Causes may include a direct effect of HIV, other cardiotropic viruses, ARV toxicity, cytokines, opportunistic infections (OIs), illicit drug use or nutritional deficiencies. In a pre-HAART series of myocardial biopsies in HIV patients, 5 of 33 stained positive for HIV, while 16 of 33 were positive for CMV using antisense riboprobes. ${ }^{20}$ The incidence of cardiomyopathy is declining post-HAART. ${ }^{21}$

Myocarditis has many causes in HIV-infected patients, though a specific diagnosis is rare. These include toxoplasmosis, tuberculosis, Cryptococcus neoformans, Aspergillus, Candida, cytomegalovirus, HSV, Mycobacterium avium-intracellulare, and HIV.22,23 In an Italian study, lymphocytic interstitial myocarditis was documented in 30 of $440(7 \%)$ of AIDS patients at autopsy. ${ }^{24}$ Again, the incidence of myocarditis has also declined in the era of effective HIV treatment.

Pericardial disease is common in HIV-infected patients. In the Prospective Evaluation of Cardiac Involvement in AIDS (PRECIA) study, before the introduction of HAART the incidence of pericardial effusion was $11 \%$ per year and it was associated with shorter survival and lower CD4 counts. ${ }^{25} \mathrm{~A}$ majority of the effusions were asymptomatic and idiopathic.

They rarely cause tamponade. ${ }^{26}$ Various OIs and malignancies have been reported to cause pericardial effusions including Kaposi's sarcoma, mycobacteria, CMV, prosthetic valve endocarditis, bacterial pericarditis caused by organisms such as Streptococcus pneumonia. ${ }^{27}$ Nocardia. ${ }^{28,29}$ lymphoma and immune reconstitution inflammatory syndrome secondary to Mycobacterium tuberculosis (TB). ${ }^{30}$ In Africa, the majority of pericardial disease in HIV infected patients is caused by TB. ${ }^{31,32}$

The incidence of pericarditis has also been decreasing since the introduction of HAART.
In a retrospective study of the incidence of cardiac disease comparing the era of mono or dual NRTI therapy versus HAART, the incidence of pericarditis had decreased from $13.5 \%$ to $3.4 \%$. Bundle branch block, atrial fibrillation, ischemia and dilated cardiomyopathy have similarly decreased.

It is unknown if the incidence of pericardial effusion has declined in the HAART era. However, the direct effects of HIV and OIs on cardiovascular tissue and the associated cardiac disease have been significantly reduced as a result of effective HIV treatment.

Thromboembolic disease has a high prevalence in HIVinfected patients, including deep vein thrombosis, pulmonary embolism, thrombotic microangiopathy and retinal venous thrombosis. A retrospective review found a rate of deep venous thrombosis ten times that of HIV-uninfected patients in the general population. ${ }^{33}$ Thromboembolic events have been found to occur at higher rates in patients with a CD4 count $<200 / \mathrm{mm} 3$ compared to those with a CD $4>200 / \mathrm{mm} 3 .^{34}$

Fultz et al. looked a 37,535 HIV-infected veterans and the same number of controls and found an increased rate of venous thromboembolism in the HIV-infected veterans, both before 1996 in the pre-HAART era and after 1996 in the HAART era after protease inhibitors became available for treatment. ${ }^{35} \mathrm{~A}$ study by Lijfering et al. found that patients with CD4 $<200 / \mathrm{mm} 3$ had higher factor VIII and fibrinogen concentrations and lower protein $\mathrm{S}$ concentrations, perhaps explaining some of the difference in thrombosis rates. ${ }^{36}$ Other factors underlying thromboembolic events in HIV infected patients are discussed in a previous section of this paper.

Pulmonary hypertension is significantly increased in HIV infected patients compared to the general population with an incidence of around $0.5 \%$ reported in 1991.37 The same incidence $(0.46 \%)$ was found in a 2008 study, thus refuting the hypothesis that the incidence had decreased since the introduction of HAART.38,39 Though HAART improved mortality in HIV-infected patients with pulmonary hypertension in a prospective study of the Swiss HIV Cohort, the median survival was poor at 2.7 years from diagnosis. ${ }^{40}$

Therefore, pulmonary hypertension portends a poorer prognosis in HIV-infected patients, despite HAART therapy. ${ }^{41}$ The rates of endocarditis are similar among HIV-infected patients with shared risk factors, such as injection drug use and non-HIV-infected individuals. HIV per se does not seem to be a risk factor for endocarditis, though mortality may be higher for those with CD4 counts under 200/mm3.42 Finally, cardiac neoplasms are a rare complication of AIDS. B cell lymphomas can be primary cardiac lymphomas or part of disseminated disease. Kaposi's sarcoma can rarely invade the heart in disseminated disease. Both of these neoplasms are more common in HIV-infected than in HIV uninfected patients.

The full extent of cardiovascular risk in treated HIVinfected patients will become more discernible as more studies continue to follow patients over time. Since the HIVinfected population is relatively young and actual cardiovascular events are infrequent, many investigators are now using surrogate measures of coronary artery disease, such as coronary artery calcium scoring, carotid intimal medial thickness and flow mediated dilatation as indirect endpoints.

In view of the high frequency of cardiac abnormalities detected in the HIV/AIDS patients in our study and well established risk of cardiovascular diseases in HIV patients, it is 
suggested that HIV-positive patients should have a careful initial and periodic cardiac evaluation and risk stratification to detect early involvement of the heart in the HIV disease.

\section{REFERENCES}

1. Klein $\mathrm{D}$, Leyden $\mathrm{W}, \mathrm{Xu} \mathrm{L}$, et al. Contribution of immunodeficiency to CHD: cohort study of HIV+ and HIVKaiser Permanente members. Presented at: 18th Conference on Retroviruses and Opportunistic Infections. Boston, MA, USA, 27 February-2 March 2011.

2. Neuhaus J, Angus B, Kowalska JD, et al. Risk of all-cause mortality associated with nonfatal AIDS and serious nonAIDS events among adults infected with HIV. AIDS 2010;24:697.

3. Antiretroviral Therapy Cohort Collaboration. Causes of death in HIV-1-infected patients treated with antiretroviral therapy, 1996-2006: collaborative analysis of 13 HIV cohort studies. Clin Infect Dis 2010;50:1387.

4. Mocroft A, Reiss P, Gasiorowski J, et al. Serious fatal and nonfatal non-AIDS-defining illnesses in Europe. J Acquir Immune Defic Syndr 2010;55:262.

5. Klein D, Hurley L, Sidney S, et al. Hospitalizations for CHD and MI among Northern California HIV+ and HIV-Men: Additional Follow-up, Changes in Practice and Framingham Risk Scores. Presented at the Thirteenth Conference on Retroviruses and Opportunistic Infections, Denver, CO February 5-8, 2006, Abstract 737.

6. Currier JS, Taylor A, Boyd F, et al. Coronary heart disease in HIV-infected individuals. J Acquir Immune Defic Syndr 2003;33:506.

7. Triant VA, Lee H, Hadigan C, Grinspoon SK. Increased acute myocardial infarction rates and cardiovascular risk factors among patients with human immunodeficiency virus disease. J Clin Endocrinol Metab 2007;92:2506.

8. Lang S, Mary-Krause M, Cotte L, et al. Increased risk of myocardial infarction in HIV-infected patients in France, relative to the general population. AIDS 2010;24:1228.

9. Freiberg MS, Chang CC, Kuller LH, et al. HIV infection and the risk of acute myocardial infarction. JAMA Intern Med 2013;173:614.

10. Lang S, Mary-Krause M, Simon A, et al. HIV replication and immune status are independent predictors of the risk of myocardial infarction in HIV-infected individuals. Clin Infect Dis 2012;55:600.

11. Helleberg M, Kronborg G, Larsen CS, et al. CD4 decline is associated with increased risk of cardiovascular disease, cancer and death in virally suppressed patients with HIV. Clin Infect Dis 2013;57:314.

12. Drozd DR, Nance RM, Delaney JAC, et al. Lower CD4 Count and higher viral load are associated with increased risk of myocardial infarction. Presented at the 21st Conference on Retroviruses and Opportunistic Infections, Boston, MA, March 3-6, 2014. Abstract \#739.

13. Strategies for Management of Antiretroviral Therapy (SMART) Study Group, El-Sadr WM, Lundgren J, et al. CD4+ count-guided interruption of antiretroviral treatment. N Engl J Med 2006;355:2283.

14. Passalaris JD, Sepkowitz KA, Glesby MJ. Coronary artery disease and human immunodeficiency virus infection. Clin Infect Dis 2000;31(3):787-97.
15. Freiberg MS, Chang CC, Kuller LH, et al. HIV infection and the risk of acute myocardial infarction. JAMA Intern Med 2013;173:614.

16. Triant VA, Lee H, Hadigan C, Grinspoon SK. Increased acute myocardial infarction rates and cardiovascular risk factors among patients with human immunodeficiency virus disease. J Clin Endocrinol Metab 2007;92(7):2506-12.

17. Obel N, Thomsen HF, Kronborg G, et al. Ischemic heart disease in HIV-infected and HIV-uninfected individuals: a population-based cohort study. Clin Infect Dis 2007;44(12):1625-31.

18. Klein D, Hurley LB, Quesenberry CP, Jr, Sidney S. Do protease inhibitors increase the risk for coronary heart disease in patients with HIV-1 infection? J Acquir Immune Defic Syndr 2002;30(5):471-7.

19. Holmberg SD, Moorman AC, Williamson JM, et al. Protease inhibitors and cardiovascular outcomes in patients with HIV-1. Lancet 2002;360(9347):1747-8.

20. Herskowitz A, Vlahov D, Willoughby S, et al. Prevalence and incidence of left ventricular dysfunction in patients with human immunodeficiency virus infection. Am J Cardiol 1993;71(11):955-8.

21. Herskowitz A, Wu TC, Willoughby SB, et al. Myocarditis and cardiotropic viral infection associated with severe left ventricular dysfunction in late-stage infection with human immunodeficiency virus. J Am Coll Cardiol 1994;24(4):1025-32.

22. Pugliese A, Isnardi D, Saini A, Scarabelli T, Raddino R, Torre D. Impact of highly active antiretroviral therapy in HIVpositive patients with cardiac involvement. J Infect 2000;40(3):282-4.

23. Mayosi BM. Contemporary trends in the epidemiology and management of cardiomyopathy and pericarditis in subSaharan Africa. Heart 2007;93(10):1176-83.

24. Sudano I, Spieker LE, Noll G, Corti R, Weber R, Luscher TF. Cardiovascular disease in HIV infection. Am Heart J 2006;151(6):1147-55.

25. Barbaro G, Di Lorenzo G, Grisorio B, Barbarini G. Cardiac involvement in the acquired immunodeficiency syndrome: a multicenter clinical-pathological study. Gruppo Italiano per lo Studio Cardiologico dei pazienti affetti da AIDS Investigators. AIDS Res Hum Retroviruses 1998;14(12):1071-7.

26. Heidenreich PA, Eisenberg MJ, Kee LL, Somelofski CA, Hollander H, Schiller NB, et al. Pericardial effusion in AIDS. Incidence and survival. Circulation 1995;92(11):3229-34.

27. Louw A, Tikly M. Purulent pericarditis due to co-infection with Streptococcus pneumoniae and Mycobacterium tuberculosis in a patient with features of advanced HIV infection. BMC Infect Dis 2007;7:12.

28. Jinno S, Jirakulaporn T, Bankowski MJ, Kim W, Wong R. Rare case of Nocardia asteroides pericarditis in a human immunodeficiency virus-infected patient. J Clin Microbiol 2007;45(7):2330-3.

29. Ramanathan P, Rahimi AR. Nocardia asteroides pericarditis in association with HIV. AIDS Patient Care STDS 2000;14(12):621-5.

30. Rapose A, Sarvat B, Sarria JC. Immune reconstitution inflammatory syndrome presenting as pericarditis and pericardial effusion. Cardiology 2007;17;110(2):142-4. 
31. Mayosi BM, Wiysonge CS, Ntsekhe M, et al. Clinical characteristics and initial management of patients with tuberculous pericarditis in the HIV era: the Investigation of the Management of Pericarditis in Africa (IMPI Africa) registry. BMC Infect Dis 2006;6:2.

32. Ntsekhe M, Hakim J. Impact of human immunodeficiency virus infection on cardiovascular disease in Africa. Circulation 2005;112(23):3602-7.

33. Saber AA, Aboolian A, LaRaja RD, Baron H, Hanna K. HIV/AIDS and the risk of deep vein thrombosis: a study of 45 patients with lower extremity involvement. Am Surg 2001;67(7):645-7.

34. Saif MW, Bona R, Greenberg B. AIDS and thrombosis: retrospective study of 131 HIV-infected patients. AIDS Patient Care STDS 2001;15(6):311-20.

35. Fultz SL, McGinnis KA, Skanderson M, Ragni MV, Justice AC. Association of venous thromboembolism with human immunodeficiency virus and mortality in veterans. Am J Med 2004;116(6):420-3.

36. Lijfering WM, Sprenger HG, Georg RR, van der Meulen PA, van der Meer J. Relationship between progression to AIDS and thrombophilic abnormalities in HIV infection. ClinChem 2008.
37. Speich R, Jenni R, Opravil M, Pfab M, Russi EW. Primary pulmonary hypertension in HIV infection. Chest 1991;100(5):1268-71.

38. Sitbon O, Lascoux-Combe C, Delfraissy JF, et al. Prevalence of HIV-related pulmonary arterial hypertension in the current antiretroviral therapy era. Am J Respir Crit Care Med 2008;177(1):108-13.

39. Barnett CF, Hsue PY, Machado RF. Pulmonary hypertension: an increasingly recognized complication of hereditary hemolytic anemias and HIV infection. JAMA 2008;299(3):324-31.

40. Zuber JP, Calmy A, Evison JM, et al. Pulmonary arterial hypertension related to HIV infection: improved hemodynamics and survival associated with antiretroviral therapy. Clin Infect Dis 2004;38(8):1178-85.

41. Taichman DB, Mandel J. Epidemiology of pulmonary arterial hypertension. Clin Chest Med 2007;28(1):1-22, vii.

42. Barbarinia G, Barbaro G. Incidence of the involvement of the cardiovascular system in HIV infection. AIDS 2003;17(Suppl 1):S46-50.

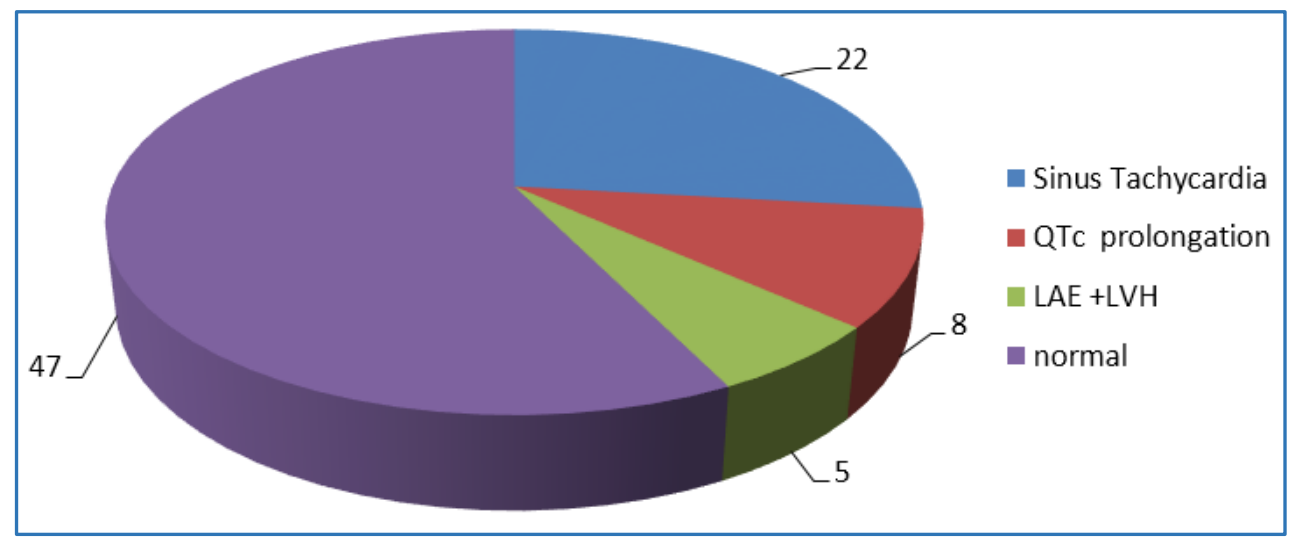

Fig. 1: ECG Findings

\begin{tabular}{|c|c|c|c|c|}
\hline CD4 Count & \multicolumn{3}{|c|}{ Echocardiographic Abnormalities } \\
\hline & \multicolumn{3}{|c|}{ Present } & \multicolumn{2}{c|}{ Absent } \\
\hline & Frequency & Percentage & Frequency & Percentage \\
\hline$<100 \mathrm{cu} \mathrm{mm}$ & 26 & 31.7 & 31 & 37 \\
\hline$>100 \mathrm{cu} \mathrm{mm}$ & 13 & 12 & 12 & 14.6 \\
\hline $\begin{array}{c}\text { Mean CD4 } \\
\text { count with SD }\end{array}$ & \multicolumn{2}{|c|}{$58.87 \pm 29.80$} & $136.53 \pm 38.80$ \\
\hline \multicolumn{4}{|c|}{ Table 1 } \\
\hline
\end{tabular}

